\title{
Thermo-economics of an irreversible solar driven heat engine
}

\author{
K. M. Pandey \& R. Deb \\ Department of Mechanical Engineering, \\ National Institute of Technology, Silchar, Assam, India
}

\begin{abstract}
Thermo-economic optimization has to be carried out for an irreversible solar driven heat engine using finite-time/finite-size thermodynamic theory. In the considered heat engine model, heat transfer from the hot reservoir is assumed to be radiation mode and the heat transfer to the cold reservoir is assumed to be convection mode. The power output per unit total cost is taken as the objective function. The steps of problem formulation are rightly performed and all valid assumptions are taken into consideration. The effects of the irreversibility parameter, economical parameter and the design parameters on the thermo-economic objective function have been investigated.

Keywords: finite-time/finite-size thermodynamics, irreversible, solar-driven heat engine, thermoeconomic optimization.
\end{abstract}

\section{Introduction}

India is endowed with abundant solar energy for about $70 \%$ of the yearly period. Solar driven heat engine systems, which consist of a solar collector and a heat engine, have a large potential for saving fossil fuel and decreasing environmental pollution. The schematic diagram of a solar driven heat engine is shown in fig.1. The energy from solar radiation is collected and utilized to generate steam to run turbines. As temperature required for steam generation is considerably high $\left(200^{\circ} \mathrm{C}\right)$, for obtaining reasonably high efficiencies, concentration type of collectors are used when steam is used as working fluid. 


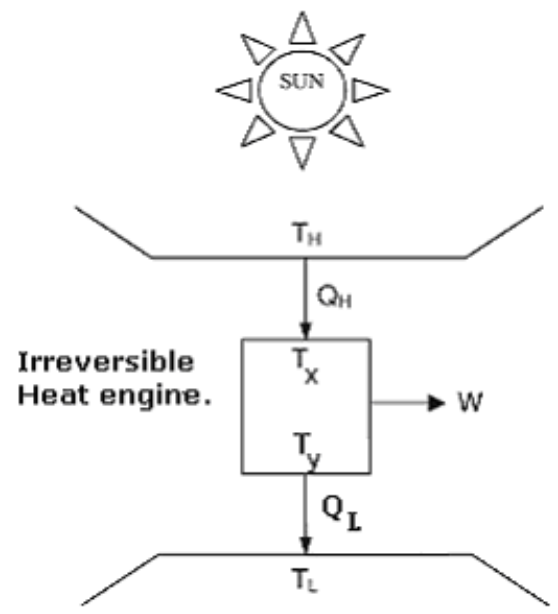

Figure 1: Schematic diagram of an irreversible solar driven heat engine.

\section{Literature review}

Power optimization studies of heat engines using finite time thermodynamics were started by Chambadal [1] and Novikov [2] and were continued by Curzon and Ahlborn [3]. The upper bound on the efficiency of a reversible engine is the so-called Carnot efficiency that is given by

$$
\eta_{\mathrm{C}}=1-\frac{T_{\mathrm{L}}}{T_{\mathrm{H}}}
$$

Firstly, Curzon and Ahlborn [3] studied the performance of an endoreversible Carnot heat engine at maximum power output. Using convective type linear heat transfer processes through finite temperature difference in both the hot and cold reservoirs, they showed that an upper limit to the endoreversible engine efficiency is

$$
\eta_{\mathrm{CA}}=1-\sqrt{\frac{T_{\mathrm{L}}}{T_{\mathrm{H}}}}
$$

The study of irreversible thermodynamic cycles has been undertaken by many researchers after Curzon and Ahlborn's work. Sahin et al. [4] studied the efficiency of a Joule-Brayton engine at maximum power density with consideration of engine size. Their results show that the efficiency at maximum power density is always greater than that presented by Curzon and Ahlborn [3]. The principle of operation of a solar thermal power plant is presented by Lund [5] in terms of finite heat transfer rates and an internally reversible heat engine by presenting some parametric equations. Medina et al. [6] extended the 
work of Sahin et al. [4] to a regenerative Joule-Brayton cycle where the optimal operating conditions at the engine were expressed in terms of the compressor and turbine isentropic efficiencies and of the heat exchanger efficiency. Many authors investigated the effects of radiative heat transfer law on the performance of heat engines. Jeter [7], De Vos and Pauwels [8] applied the Stefan-Boltzmann thermal radiation law to the performance analysis of solar powered energy systems. De Vos [9], Chen and Yan [10] and Gordon [11] discussed the effect of a class of heat transfer laws (linear and non-linear radiation) on the performance of endoreversible cycles. They revealed the dependence of performance on the heat-transfer law and the heat-transfer coefficient and derived a universal expression for different common heat transfer laws and concluded that the value of maximum power depends on both the source temperatures and the heattransfer coefficient. Gordon [12] illustrated that the optimal operating temperature for solar-driven heat engines and solar collectors is relatively insensitive to the engine design point. Bejan $[13,14]$ performed a power optimization study for a solar driven power plant model. He examined the optimal design parameters and optimal distribution of the heat transfer areas at maximum power output conditions. In recent years, the performance of a solar driven heat engine using the technique of finite time thermodynamic analysis has been investigated. In these studies, the objective functions chosen for optimization are usually power output. Chen [15] investigated the optimal performance and design parameters for solar driven heat engine consisting of a solar collector and a heat engine. In the study, he determined the optimum operating temperatures of the working fluid and solar collector. Goktun et al. [16] investigated the design parameters of an endoreversible radiative heat engine at maximum power output conditions. As a major result, they showed that the ratio of the cold to the hot reservoir temperature must be less than 0.2 for an optimal design. The work carried out by Goktun et al. [16] for an endoreversible radiative heat engine model has been extended to an irreversible radiative heat engine model by Ozkaynak [17]. He obtained the design parameters at maximum power output for radiative and convective boundary conditions. He also discussed the effects of internal and external irreversibility parameters on the maximum power output and thermal efficiency at maximum power conditions. Badescu [18] proposed a model of a space power station composed of an endoreversible Carnot heat engine driven by solar energy. He obtained the maximum power output and the optimum ratio between the solar collector and radiator areas. Erbay and Yavuz [19] performed an analysis of an endoreversible Carnot heat engine with the consideration of combined radiation and convection heat transfer between the working fluid and hot and cold heat reservoirs. They showed that the power output is strongly dependent on the temperature and emittance ratios of the heat reservoirs. Badescu et al. [20] performed a power optimization for solar driven endoreversible Carnot heat engine model. They obtained optimum solar collector surface area and temperature under maximum power conditions. Sahin [21] carried out optimization study based on maximum power criterion for an endoreversible solar driven heat engine with radiation mode heat transfer from the hot reservoir and convection mode to the cold 
reservoir. He also developed his model in [21] by considering the collective role of radiation and convection heat transfer from hot reservoir [22]. In these studies, he investigated the optimal working fluid temperatures and the thermal efficiency at maximum power conditions. He also discussed the effects of the ratios of the reservoir temperatures and the heat transfer coefficients on the optimal performances. Koyun [23] carried out a comparative performance analysis based on maximum power and maximum power density criteria for a solar-driven heat engine with external irreversibilities. He compared the optimal performances and design parameters at the maximum power and power density conditions. Sahin and Kodal [24] have recently reported a new performance analysis based on an objective function defined as the power output per unit total cost. Using this performance optimization criterion, they performed a finite-time thermoeconomic optimization for endoreversible [24] and irreversible [25] heat engines with linear heat transfer modes. They investigated the optimal performances and design parameters under the maximum thermoeconomic objective function conditions and then they examined the effects of technical and economical parameters on the global and optimal performances. Sahin et al. [27] have recently carried out thermoeconomic optimization for an endoreversible solar driven heat engine using finite time/finite size thermodynamic theory. They investigated the effects of the technical and economical parameters on the thermoeconomic performances. In this paper, the finite-time thermoeconomic optimization technique introduced by Kodal and Sahin [25] for an irreversible heat engine model with linear heat transfer modes has been applied to a solar driven irreversible heat engine model to perform thermo-economic optimization.

\section{Aim of the work}

The aim of this work is to perform thermo-economic optimization of an irreversible solar driven heat engine using finite-time thermodynamics.

\subsection{Theoretical model}

Solar powered heat engine is considered to operate according to the Rankine cycle given in Fig. 2. The considered Rankine cycle operates between a heat source of temperature $T_{H}$ and a heat sink of temperature $T_{L}$. In order to simplify the analysis, the Rankine cycle (1-2-3-4-5-1) can be modified by using an entropic average temperature defined by Khaliq [26] to a Carnot cycle (1-a-b-51). Since the area under the process 2-3-4 in the T-S diagram of Fig. 2 represents the amount of heat added to the Rankine cycle, we can make this area equal to the area under the horizontal line with an entropic average temperature of heat addition. The entropic average temperature can be written as,

$$
T_{X}=\Delta Q / \Delta s=\left(h_{4}-h_{2}\right) /\left(s_{4}-s_{2}\right)
$$



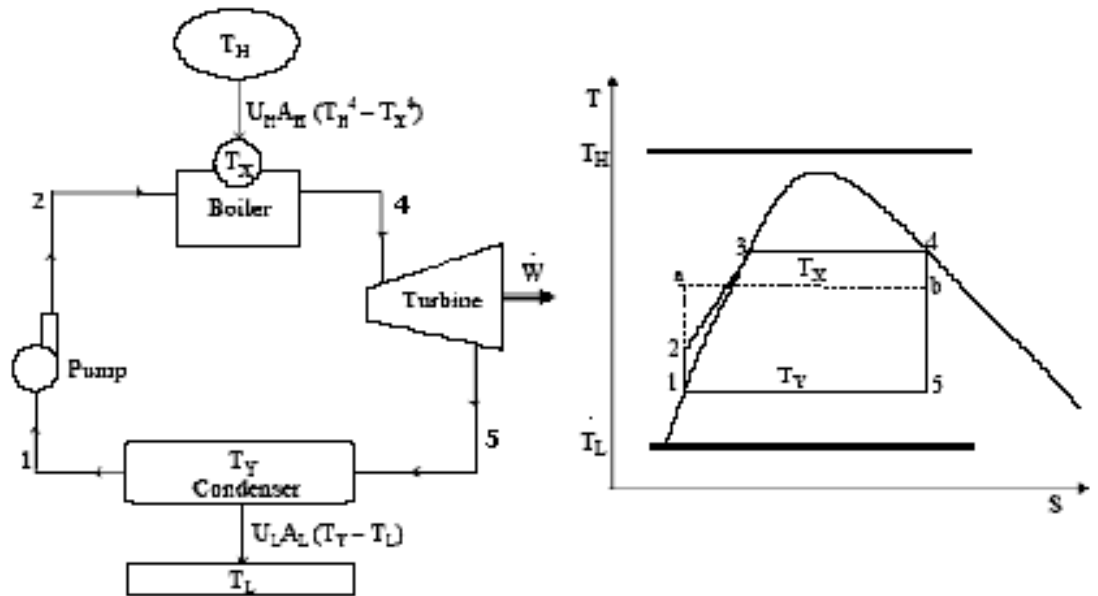

Figure 2: The components of a solar driven heat engine and its T-S diagram.

\section{Problem formulation}

The irreversible Carnot-type solar driven heat engine operating between temperature limits $\left(\mathrm{T}_{\mathrm{H}}\right.$ and $\left.\mathrm{T}_{\mathrm{L}}\right)$ is shown in fig. 3 .

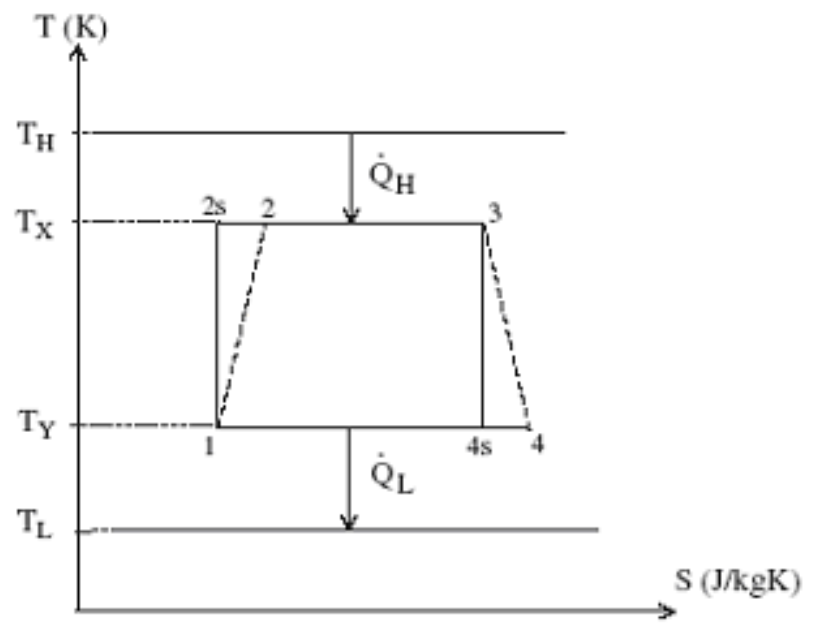

Figure 3: T-S diagram of a Carnot-type solar driven heat engine.

Heat transfer from the hot reservoir is assumed to be radiation dominated and the heat flow rate $\mathrm{Q}_{\mathrm{H}}$ from the hot reservoir to the heat engine can be written as

$$
\dot{Q}_{H}=U_{H} A_{H}\left(T_{H}^{4}-T_{X}^{4}\right)
$$


where $A_{H}$ is the heat transfer area of the hot side heat exchanger. $U_{H}$ is the hot side heat transfer coefficient. On the other hand, convection heat transfer is assumed to be the main mode of heat transfer to the low temperature reservoir and therefore the heat flow rate $\mathrm{Q}_{\mathrm{L}}$ from the heat engine to the cold reservoir can be written as

$$
\dot{Q}_{L}=U_{L} A_{L}\left(T_{Y}-T_{L}\right)
$$

where $U_{L}$ is the cold side heat transfer coefficient and $A_{L}$ is the heat transfer area of the cold side heat exchanger. From the first law of the thermodynamics the net power output of the solar driven heat engine is

$$
\dot{W}=\dot{Q}_{H}-\dot{Q}_{L}
$$

Using eqns (1) and (2) in (3), we get

$$
\dot{W}=U_{L} A_{L}\left[\mu \frac{T_{H}^{4}-T_{X}^{4}}{T_{H}^{3}}-\left(T_{Y}-T_{L}\right)\right]
$$

where $\mu$ is the product of ratio of heat transfer areas (R) and the heat conductance parameter $(\delta)$ and is defined as

$$
\mu=\mathrm{R} \times \delta
$$

and

$$
\mathrm{R}=\mathrm{A}_{\mathrm{H}} / \mathrm{A}_{\mathrm{L}}
$$

and

$$
\delta=\frac{U_{H}}{U_{L}} T_{H}^{3}
$$

From the second law of thermodynamics for an irreversible cycle, the change in the entropies of the working fluid for heat addition and heat removing processes yields,

$$
\oint \frac{\delta \dot{Q}}{T}=\frac{\dot{Q}_{H}}{T_{X}}-\frac{\dot{Q_{L}}}{T_{Y}}<0
$$

One can rewrite the inequality in eqn (8) as

$$
\frac{\dot{Q}_{H}}{T_{X}}=I \frac{\dot{Q_{L}}}{T_{Y}}, 0<I<1 .
$$

where I is irreversibility parameter. 
With the above definition I becomes

$$
I=\frac{\dot{Q}_{H} T_{Y}}{\dot{Q}_{L} T_{X}}=\frac{T_{X}\left(s_{3}-s_{2}\right) T_{Y}}{T_{Y}\left(s_{4}-s_{1}\right) T_{X}}=\frac{s_{3}-s_{2}}{s_{4}-s_{1}}
$$

From eqns (1), (2) and (9), we get

$$
T_{Y}=\frac{T_{L}}{1-\frac{\mu\left(T_{H}^{4}-T_{X}^{4}\right)}{I T_{X} T_{H}^{3}}}
$$

The thermal efficiency of the irreversible heat engine is

$$
\eta=1-\frac{\dot{Q_{L}}}{\dot{Q}_{H}}=1-\frac{T_{Y}}{I T_{X}}
$$

In thermoeconomic analysis, an objective function is defined as power output per unit total cost in order to account for both investment and fuel costs. In this study, the objective function has been defined as the power output per unit investment cost due to no fuel consumption cost in a solar driven heat engine. In order to optimize power output per unit total cost, the objective function is defined as

$$
\mathrm{F}=\mathrm{W} / \mathrm{Ci}
$$

where $C_{i}$ refers to annual investment cost. The investment cost of the plant is assumed to be proportional to the size of the plant. The size of the plant can be taken proportional to the total heat transfer area. Thus, the annual investment cost of the system can be given as

$$
C_{i}=\left(h A_{H}+l A_{L}\right)
$$

where the investment cost proportionality coefficients for hot and cold sides $h$ and 1 are equal to the capital recovery factor times investment cost per unit heat transfer area. Substituting eqns (4) and (14) into eqn (13), we obtain

$$
F=\frac{\left[\mu \frac{\left(T_{H}^{4}-T_{X}^{4}\right)}{T_{H}^{3}}-\left(T_{Y}-T_{L}\right)\right]}{l\left[\left(\frac{k}{1-k}\right) R+1\right]} U_{L}
$$

where $\mathrm{k}$ is relative investment cost parameter of the hot side heat exchanger and defined as 
430 Advanced Computational Methods in Heat Transfer IX

$$
k=h /(h+l)
$$

Dimensionless thermo economic objective function is defined as

$$
\bar{F}=l F /\left(U_{L} T_{L}\right)
$$

Dimensionless power output is defined as

$$
\overline{\dot{W}}=\dot{W} /\left(U_{L} A_{L} T_{L}\right)
$$

Using eqn (15) in (17), we get

$$
\bar{F}=\frac{\left[\mu \frac{\left(T_{H}^{4}-T_{X}^{4}\right)}{T_{H}^{3}}-\left(T_{Y}-T_{L}\right)\right]}{\left[\left(\frac{k}{1-k}\right) R+1\right] T_{L}}
$$

Using eqn (4) in (18), we get

$$
\dot{\bullet}=\left[\mu \frac{\left(T_{H}^{4}-T_{X}^{4}\right)}{T_{L} T_{H}^{3}}-\frac{\left(T_{Y}-T_{L}\right)}{T_{L}}\right]
$$

Using eqn (11) in (12), we get

$$
\eta=1-\frac{T_{L} T_{H}^{3}}{I T_{X} T_{H}^{3}-\mu\left(T_{H}^{4}-T_{X}^{4}\right)}
$$

Putting $\theta=T_{X} / T_{H}$ and $\tau=T_{H} / T_{L}$ in eqn (19), (20) and (21) we get

$$
\begin{gathered}
\bar{F}=\left[\mu \tau\left(1-\theta^{4}\right)-\frac{\mu\left(1-\theta^{4}\right)}{I \theta-\mu\left(1-\theta^{4}\right)}\right] /\left[\left(\frac{k}{1-k}\right) R+1\right] \\
\bar{W}=\mu \tau\left(1-\theta^{4}\right)-\frac{\mu\left(1-\theta^{4}\right)}{I \theta-\mu\left(1-\theta^{4}\right)} \\
\eta=\frac{I \tau \theta-\mu \tau\left(1-\theta^{4}\right)-1}{I \tau \theta-\mu \tau\left(1-\theta^{4}\right)}
\end{gathered}
$$

To maximize the dimensionless thermoeconomic objective function, eqn (22) is differentiated with respect to $\theta$ and the resulting derivative is equated to zero. After a lengthy calculation, it is found that the optimum value of $\theta$ must satisfy the following equation 


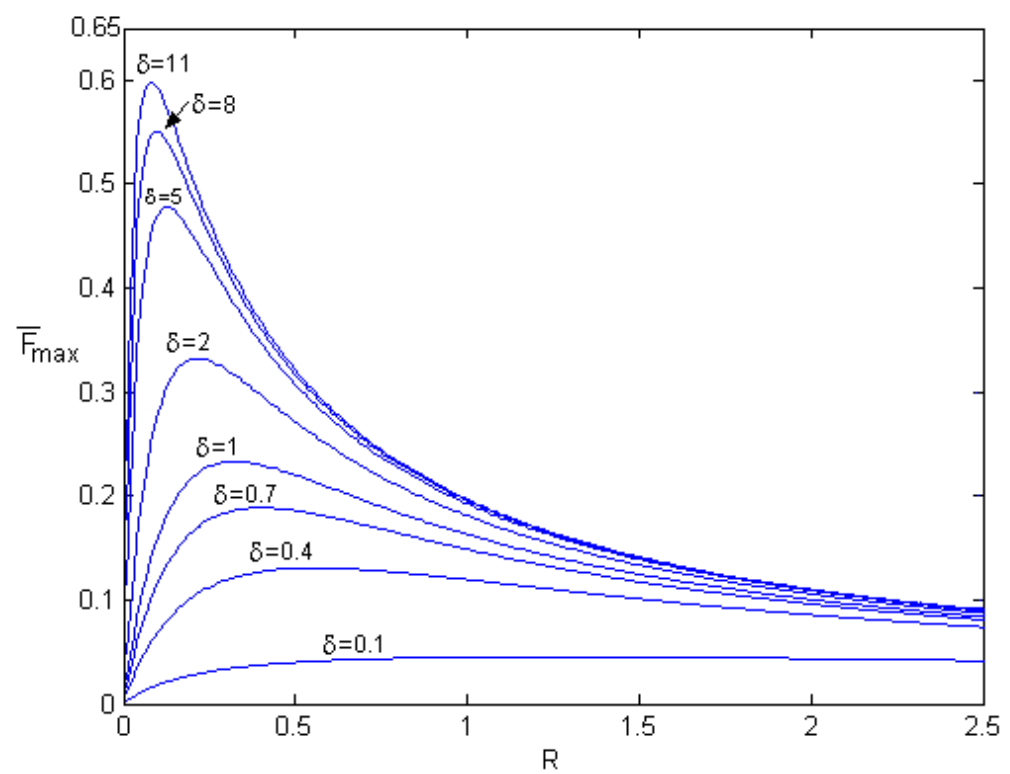

(a)

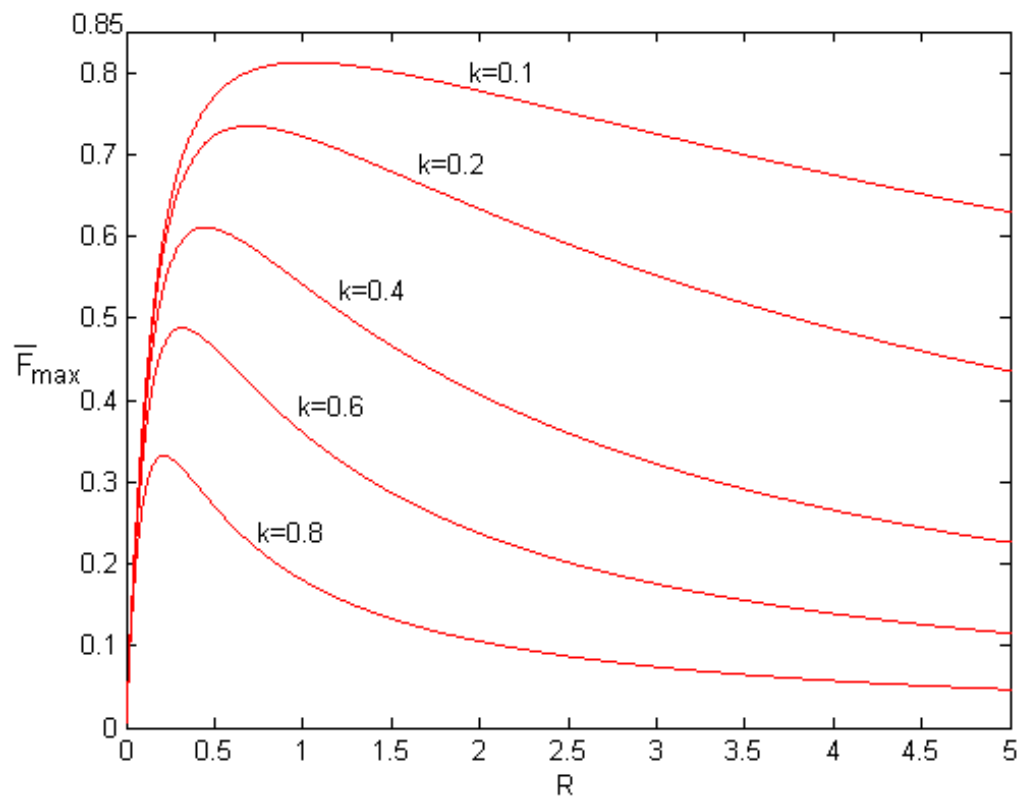

(b)

Figure 4: Variation of the maximum thermoeconomic objective function with respect to $\mathrm{R}$ for various (a) $\delta$ values $(\mathrm{I}=0.8, \mathrm{k}=0.8, \tau=5)$ (b) $\mathrm{k}$ values $(\mathrm{I}=0.8, \tau=5, \delta=2)(\mathrm{c}) \tau$ values $(\mathrm{I}=0.8, \delta=2, \mathrm{k}=0.8)(\mathrm{d}) \mathrm{I}$ values $(\tau=5$, $\delta=2, \mathrm{k}=0.4$ ). 
432 Advanced Computational Methods in Heat Transfer IX

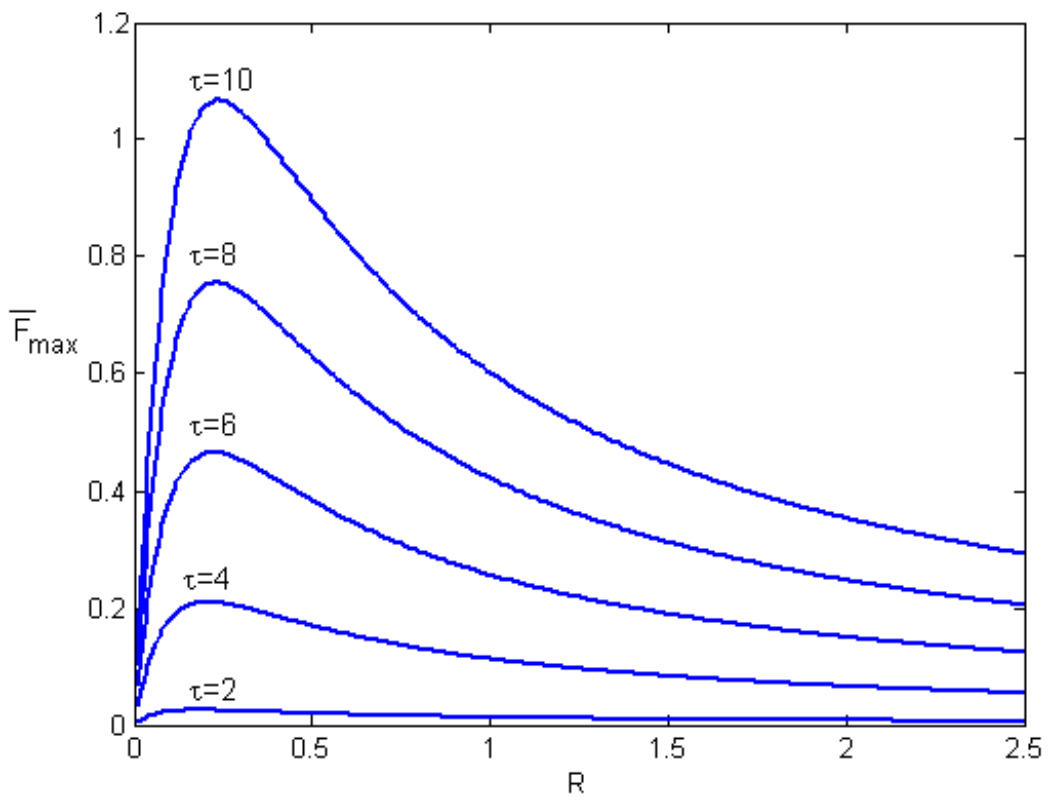

(c)

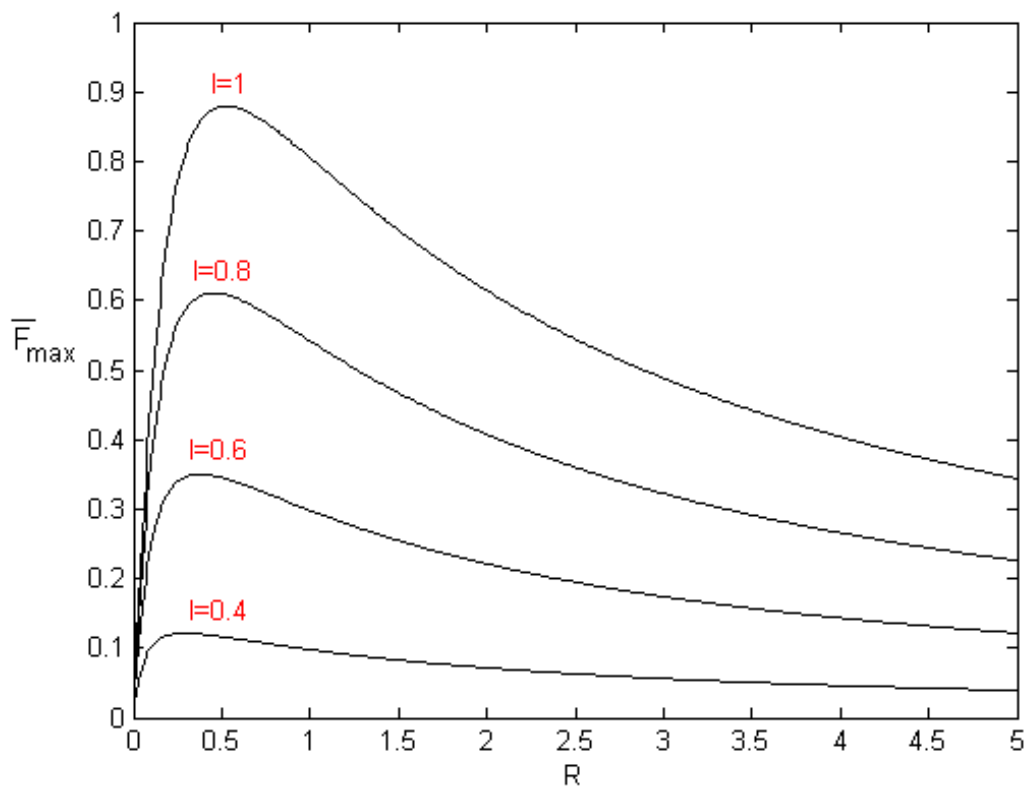

(d)

Figure 4: Continued. 


$$
4 \mu^{2} \tau \theta^{11}+8 \mu \tau I \theta^{8}-8 \mu^{2} \tau \theta^{7}+4 \tau I^{2} \theta^{5}-(8 \mu \tau I+3 I) \theta^{4}+4 \mu^{2} \tau \theta^{3}-I=0
$$

Solution of eqn (25) can be done numerically.

\section{Results and discussion}

The variations of maximum thermoeconomic objective function with respect to different technical and economical parameters are shown in figure 4.From the figures, it is clear that the optimal value of ratio of heat transfer areas(R) for which the maximum value of thermo-economic objective function becomes maximum depends on $\delta, \mathrm{k}, \mathrm{I}$ and $\tau$ parameters. It is seen from figure 4(a) that the optimal value of ratio of heat transfer areas (R) decreases with increase in the value of heat conductance parameter $(\delta)$. Also with increase in the value of $\delta$, the optimal value of objective function also increases. On increasing the value of $\delta$ beyond unity, the optimal objective function increases sharply for $\mathrm{R}$ lying below 1 .

From figure 4(b), it can be observed that the optimal value of ratio of heat transfer areas $(\mathrm{R})$ decreases with increase in the value of economical parameter (k). Also the optimal value of objective function decreases with increase in the value of $\mathrm{k}$.

From figure $4(\mathrm{c})$, it is seen that the maximum value of thermoeconomic objective function increases sharply with increase in the value of $\tau$ for the value of $\mathrm{R}$ lying below 0.5 .Also the optimal value of $\mathrm{R}$ is not get affected with increase in the value of $\tau$.

From figure 4(d), it observed that the optimal value of $\mathrm{R}$ decreases slightly with decreasing I. Also there is severe fall in the value of maximum objective function with slight decrease in the value of $\mathrm{I}$. Also the optimal value $\mathrm{R}$ lies below unity.

\section{Conclusion}

A thermo-economic optimization has been carried out for an irreversible solar driven heat engine. The objective function has been defined as the ratio of power output to the total investment cost for setting up the plant. The effects of irreversibility parameter, economical parameter and the design parameters on the thermo-economic objective function have been investigated. By optimizing the objective function the optimum ranges for various parameters have been determined.

\section{References}

[1] Chambadal P. Les Centrales Nuclearies. Paris: Armond Colin; 1957. p. 41-58. 
[2] Novikov II. The efficiency of atomic power stations (a review). Atom Energy 1957; 3(11):409.

[3] Curzon Fl, Ahlborn B. Efficiency of a Carnot engine at maximum power output. Am J Phys 1975; 43:22-4.

[4] Sahin B, Kodal A, Yavuz H. Efficiency of a Joule-Brayton engine at maximum power density. Journal of Physics, D: Applied Physics 1995; 28:1309-13.

[5] Lund KO. Applications of finite-time thermodynamics to solar power conversion. In: Sieniutycz S, Salamon P, editors. Finite-time thermodynamics and thermoeconomics. London: Taylor \& Francis, 1990. p. 121.

[6] Medina A., Roco J.M.M., Hernandez A.C., Regenerative gas turbines at maximum power density conditions, J. Phys. D: Appl. Phys. 29 (1996) 2802-2805.

[7] Jeter S. Maximum conversion efficiency for the utilization of direct solar radiation. Sol Energy 1981; 26:231-6.

[8] De Vos A, Pauwels H. On the thermodynamic limit of photovoltaic energy conversion. J Appl Phys 1981; 25:119-25.

[9] De Vos A. Efficiency of some heat engines at maximum power conditions. Am J Phys 1985; 5:570-3.

[10] Chen L, Yan Z. The effect of heat transfer law on performance of a twoheat-source endoreversible cycle. J Chem Phys 1989; 9:3740-3.

[11] Gordon JM. Observations on efficiency of heat engines operating at maximum power. Am J Phys 1990; 58:370-5.

[12] Gordon JM. On optimized solar-driven heat engines. Sol Energy 1988; 40:457-61.

[13] Bejan A. Advanced engineering thermodynamics. New York: Wiley; 1988.

[14] Bejan A. Heat transfer. New York: Wiley; 1993.

[15] Chen J. Optimization of a solar-driven heat engine. J Appl Phys 1992; 72:3778-80.

[16] Goktun S, Ozkaynak S, Yavuz H. Design parameters of a radiative heat engine. Energy 1993; 18:651-5.

[17] Ozkaynak S. Maximum power operation of a solar-powered heat engine. Energy 1995; 20:715-21.

[18] Badescu V. Optimum design and operation of a dynamic solar power system. Energy Convers Manage 1996; 37:151-60.

[19] Erbay LB, Yavuz H. An analysis of an endoreversible heat engine with combined heat transfer. J Phys D: Appl Phys 1997; 30:2841-7.

[20] Badescu V, Popescu G, Feidt M. Model of optimized solar heat engine operating on Mars. Energy Convers Manage 1999; 40:1713-21.

[21] Sahin AZ. Optimum operating conditions of solar driven heat engines. Energy Convers Manage 2000; 41:1335-43.

[22] Sahin AZ. Finite-time thermodynamic analysis of a solar driven heat engine. Energy Int J 2001; 2:122-6. 
[23] Koyun A. Performance analysis of a solar driven heat engine with external irreversibilities under maximum power and power density condition. Energy Convers Manage 2004; 45:1941-7.

[24] Sahin B, Kodal A. Performance analysis of an endoreversible heat engine based on a new thermoeconomic optimization criterion. Energy Convers Manage 2001; 42:1085-93.

[25] Kodal A, Sahin B. Finite size thermoeconomic optimization for irreversible heat engines. Int J Therm Sci 2003; 42:777-82.

[26] Khaliq A. Finite-time heat transfer analysis and generalized power optimization of an endoreversible Rankine heat-engine. Appl Energy 2004; 79:27-40.

[27] Sahin B., Ust Y., Yilyaz T., Akcay I.H. Thermoeconomic optimization of a solar driven heat engine. Renewable Energy xx (2005) 1-10. 